\title{
A BASIC REVIEW ON MICRO EMULSION AS A DRUG DELIVERY SYSTEM.
}

\section{Alfisha Saifi , Kapil Kumar, Deepak Teotia}

Department of Pharmaceutics, Global Institute of Pharmaceutical Education \& Research, Kashipur-244713, Uttarakhand, India

\author{
Article Info: Received 25 July 2019; Accepted 14 September. 2020 \\ DOI: https://doi.org/10.32553/jbpr.v9i5.798 \\ Corresponding author: Alfisha Saifi \\ Conflict of interest statement: No conflict of interest
}

\section{ABSTRACT:}

Lipid dosage forms are attractive delivery systems for hydrophobic drug molecules. Emulsion is one of the popular system since many decades. Pharmaceutical applications of emulsions widened especially after micro emulsion emergence. Now a day Microemulsion is an emerging trade and having worldwide importance in a variety of technological applications. These applications include enhanced oil recovery, combustion, cosmetics, pharmaceuticals, agriculture, metal cutting, lubrication, food, enzymatic catalysis, organic and bio-organic reactions, chemical synthesis of nanoparticles etc. This review article deals with feature and application of microemulsion. a brief introduction and definition, structure, type, formation characteristics, stability, phase behavior and the effect of additives, pressure, temperature on the phase behavior of microemulsion . In addition to oral and intravenous delivery, they are amenable for sustained and targeted delivery through ophthalmic, dental, pulmonary, vaginal and topical routes. Microemulsions are experiencing a very active development as reflected by the numerous publications and patents being granted on these systems. They have been used to improve the oral bioavailability of various poorly soluble drugs including cyclosporine.

Keywords: Microemulsion, Self micro emulsifying system, poor soluble, thermodynamically stable, Sustained \& Controlled release, Drug delivery.

\section{INTRODUCTION}

Emulsions are dispersions made up of two immiscible liquid phases which are mixed using mechanical shear and surfactant. Particle size of this conventional emulsion grows continuously with time and hence finally separation occurs at gravitational force thus these emulsions are thermodynamically unstable ${ }^{1}$.

Micro emulsion is Dispersion system made of water, oil, and surfactant(s) that is anisotropic and thermodynamically stable with a diameter varying approximately from 1 to $100 \mathrm{~nm}$, usually 10 to $50 \mathrm{~nm}$. Micro emulsions are clear, thermodynamically stable, anisotropic liquid mixtures of oil, water and surfactant, frequently combined with a co surfactant. The aqueous phase may contain active pharmaceutical ingredients along with excipients. The "oil" is a complex mixture of different hydrocarbons and olefins. The term of micro emulsion coined to a mixture with at least three components, i.e., an oily phase, an aqueous phase and a surface active species (surfactants) ${ }^{2}$. Sometimes the forth component i.e., co-surfactant can be present. Depending on the ratios between the components, in the two extremes the microstructure, the micro emulsions vary from a very tiny water droplets dispersed in oil phase (w/o micro emulsion) to oil droplets dispersed in water phase (o/w micro emulsion). The microstructure of the mixture changes continuously from one to another, named, from a spherical to cylindrical, tubular and interconnected oil and water phases separated with a very thin layer of surfactant molecules, in the middle, that is defined as discontinues micro emulsion. The micro emulsions of each kind are thermodynamically stable and transparent solutions. There are main differences between emulsions and micro emulsions in terms of structure and stability. In contrast to the micro emulsions, the emulsions are unstable systems and without agitation their phase may separate out. The other difference is that in the size of droplets in emulsions \& microemulsion, it ranges in micrometers for emulsion, while in micro emulsions the size of micelles are in the range of 5-100 nm, depending on the some parameters such as type of surfactant used ,its concentration, \& the extent of dispersed. Hence, sometimes the micro emulsion term is misleading, because it doesn't reflect the size of dispersed phase droplets in the system which, are in the nanometer range. Depending on the type of the surfactants employed in the preparation of the micro emulsion, another important parameter that affects the main 
characteristics of a micro emulsion is the presence of electrolytes in the aqueous phase ${ }^{3}$.

\section{Theories: Interfacial theory}

It is also called as mixed film or dual film theory. Surfactant and co-surfactant together forms complex film at the oil water interface and thus creates generation of micro emulsion droplets ${ }^{4}$.

\section{Solubilization theory}

This theory assumes that swollen micellar system forms in the form of micro emulsion. Oil solubilised due to normal micelle formation and water solubilised by reverse micelle formation.

\section{Thermodynamic theory}

When interfacial tension between two immiscible phases reduces to zero, causes spontaneous formation of micro emulsions and formed negative free energy helps to make emulsion thermodynamically stable ${ }^{5}$.

\section{History and Terminology:}

Micro emulsions were not really recognized in earlier 70 s until the work of Hoar and Schulman in 1943, that reported a spontaneous emulsion of water and oil on addition of a strong surface-active agent. Microemulsions are isotropic, thermodynamically stable transparent (or translucent) systems of oil, water and surfactant, frequently in combination with a cosurfactant with a droplet size usually ranging in 20-200 $\mathrm{nm}$. These homogeneous systems, can be prepared with a wide range of surfactant concentration and oil to water ratio, with all fluids of low viscosity. Microemulsions are potent drug delivery system that shows favorable properties like long shelf-life due to thermodynamically stability, easy formation or almost spontaneous formation with zero interfacial tension, optically active, ability to be sterilized by filtration, high surface area , high solubilization capacity and with very small droplet size.

There has been much debate about the word "micro emulsion" to describe such systems. Although not systematicallyterm is used in today's era, but still some prefer the names "micellar emulsion" or "swollen micelles" ${ }^{\prime \prime}$.

Interest in micro emulsions really stepped up in the earlier 1980's when it was recognized that this kind of systems can improve oil recovery and when oil prices reached higher levels.

The drug delivery system should be mild and biocompatible, the choice of excipients is, however, limited. Also a large amount of surfactant is required for the formation of microemulsion which is undesirable and expensive. Therefore, a proper selection of the components and their concentration used is esssential for a wider acceptability and temperature stability ${ }^{7}$.

SMEDDS:A self microemulsifying drug delivery system (SMEDDS) is an anhydrous system of microemulsions. It has also been referred as microemulsion preconcentrate by some researchers, as It is composed of oil, surfactant and cosurfactant and has the ability to form o/w microemulsion when dispersed in aqueous phase under gentle agitation. The agitation required for the self-emulsification comes from stomach and intestinal motility ${ }^{8}$.

\section{Advantages:}

- Thermodynamically stable and require minimum energy for formation. compatibility in production.

- Enhanced drug solubilization and improved bioavailability.

- Micro emulsion is having wide applications in colloidal drug delivery systems.

- Drug targeting and controlled release.

\section{Disadvantages:}

- Use of large concentration of surfactant and cosurfactant necessary for stabilizing the nanodroplets.

- Limited solubilizing capacity.

- The surfactant must be nontoxic \& inert.

- Microemulsion stability is influenced by environmental parameters such as temperature and $\mathrm{pH}$. These parameters change upon microemulsion delivery to patients.

Important Characteristics of Microemulsions ${ }^{9}$ :

- Particle size 10-100 nm.

- Thermodynamically stable (long shelf-life).

- Optically clear.

- High surface area.

- Small droplet size.

- Enhanced drug solubilization.

- Easy formation.

- Ability to be sterilized by filtration.

- High solubilization capacity for hydrophilic and lipophilic drugs.

- Improved drug delivery.

- Controlled released.

- Biocompatible.

Table 1: Difference between emulsion \& micro-emulsion

\begin{tabular}{ll}
\hline EMULSION & MICROEMULSION \\
\hline $\begin{array}{l}\text { Emulsions consist of roughly spherical } \\
\text { droplets of one phase dispersed into } \\
\text { the other. }\end{array}$ & $\begin{array}{l}\text { They constantly evolve between } \\
\text { various structures ranging from } \\
\text { droplet like swollen micelles to bi- } \\
\text { continuous structure }\end{array}$ \\
\hline Thermodynamically unstable & Thermodynamically stable \\
\hline Inefficient molecular packing & Efficient molecular packing \\
\hline High interfacial tension & Ultra low interfacial tension \\
\hline They are lyophobic & They between lyophobic and lyophilic \\
& colloids \\
\hline Droplet diameter: $>500 n m$ & $10-100$ nm \\
\hline Cloudy colloidal system & Optically transparent \\
\hline High viscosity & Low viscosity \\
\hline
\end{tabular}




\section{STRUCTURE OF MICROEMULSION:}

The dispersed mixture of oil, water and surfactants is able to form a wide variety of structures and phases depending upon the proportions of the components. Theflexibility of the surfactant film is an important factor. A flexible surfactant film shall enable the existence of several different structures like droplet shapes, aggregates and bicontinuous structures, and therefore broadening the range of microemulsion. A very rigid surfactant film shall not be enable existence of bicontinuous structures which will delay the range of existence. Besides microemulsions, structural evaluations can reveal the existence of regular emulsions, anisotropic crystalline hexagonal or cubic phases, and lamellar structures depending on the ratio of the components. The internal structure of a microemulsion is very important for the diffusivity of the phases, and thereby also for the diffusion of a drug in the respective phases of dispersion medium. Research have been made to understand the complicated phase behavior of microemulsion and its various microstructures encountered in the systems.

Micro emulsions usually exhibit low viscosities and Newtonian flow characteristics. Their flow remains constant when subjected to different shear rates. Discontinuous formulations may show some nonNewtonian flow and plasticity. Micro emulsion viscosity is close to that of water, even at high droplet concentrations. The structure of microemulsion constantly changes, making them very dynamic systems with reversible droplet coalescence. Various techniques are applied to characterize different properties of micro emulsions. Light scattering, X-ray diffraction, ultracentrifugation, electrical conductivity, and viscosity measurements are some of them ${ }^{10}$.

\section{CLASSIFICATION OF MICRO EMULSION:}

According to Winsor, there are four types of micro emulsion phases exists in equilibrium, these phases are referred as Winsor phases.10-13 they are:

- Winsor I (two phase system): upper oil layer exists in equilibrium with lower (o/w) micro emulsion phase

- Winsor II (two phase system): the upper (w/o) micro emulsion exists in equilibrium with lower excess water.

- Winsor III (three phase system): middle bicontinuous phase of $\mathrm{o} / \mathrm{w}$ and $\mathrm{w} / \mathrm{o}$ called) exists in equilibrium with upper phase oil and lower phase water.

- Winsor IV (single phase system): it forms homogenous mixture of oil, water and surfactant ${ }^{11}$.

\section{PREPARATION METHOD OF MICROEMULSION:}

Following are the method used for the preparation of the micro emulsion:

1) Phase inversion method:

\section{2) Phase titration method:}

Phase Inversion Method:

Phase inversion of microemulsions occurs upon addition of excess of the dispersed phase or in response to temperature. During phase inversion drastic physical changes occur including changes in particle size that can affect drug release both in vivo and in vitro. For nonionic surfactants, this can be achieved by changing the temperature of the system, forcing a transition from an o/w microemulsion at low temperatures to a w/o microemulsion at higher temperatures (transitional phase inversion). During cooling, the system crosses a point of zero spontaneous curvature and minimal surface tension, promoting the formation of finely dispersed oil droplets. This method is referred to as phase inversion temperature (PIT) method $^{12}$.

Instead of the temperature, other parameters such as salt concentration or $\mathrm{pH}$ value may be considered as well instead of the temperature alone. Additionally, a transition in the spontaneous radius of curvature can be obtained by changing the water volume fraction. By successively adding water into oil, initially water droplets are formed in a continuous oil phase.

Increasing the water volume fraction changes the spontaneous curvature of the surfactant from initially stabilizing a w/o microemulsion to an o/w microemulsion at the inversion locus. Microemulsions can be prepared by controlled addition of lower alkanols (butanol, pentanol and hexanol) to milky emulsions to produce transparent solutions comprising dispersions of either water-in-oil $(w / o)$ or oil-in-water $(o / w)$ in nanometer or colloidal dispersions ( $100 \mathrm{~nm}$ ).

\section{Phase Titration Method:}

Micro emulsions are prepared by the spontaneous emulsification method (phase titration method) and can be depicted with the help of phase diagrams. Construction of phase diagram is a useful approach to study the complex series of interactions that can occur when different components are mixed. Micro emulsions are formed along with various association structures (including emulsion, micelles, lamellar, hexagonal, cubic, and various gels and oily dispersion) depending on the chemical composition and concentration of each component. The understanding of their phase equilibrium and demarcation of the phase boundaries are essential aspects of the study. As quaternary phase diagram (four component system) is time consuming 
and difficult to interpret, pseudo ternary phase diagram is often constructed to find the different zones including micro emulsion zone, in which each corner of the diagram represents $100 \%$ of the particular component. The region can be separated into w/o or o/w micro emulsion by simply considering the composition that is whether it is oil rich or water rich ${ }^{13}$.

\section{COMPOSITION:}

The Major component in micro emulsion system are-

1) Oil phase

2) Surfactant (primary surfactant)

3) Co-surfactant (secondary surfactant)

4) Co-solvent

Table 2: Component of Microemulsion System

\begin{tabular}{ll}
\hline Component & Example \\
\hline Oil & 1) saturated fatty acid- lauric acid, \\
& carpic acid \\
& 2) unsaturated fatty acid-oleic acid, \\
& linolic acid, linolenic acid \\
& 3) fatty acid ester-ethyl or methyl ester \\
& of lauric, oleic acid and myristic acid \\
\hline Surfactant & 1) polyoxyethylene/polysorbate/tween \\
& 20,40,60,80 \\
& 2) sorbitan monolaurate, eggs lecithin \\
\hline 3) sodium dodecyl sulphate \\
\hline 1) ethanol, proranol, butanol, \\
\\
isopropanol, pentanol, hexanol \\
2)polyoxyethylene-10-oelyl ether \\
3)sodium monohexyl phosphate \\
4)cinnamic alcohol, cinamic alcohol \\
\hline
\end{tabular}

\section{FACTORS AFFECTING MICROEMULSION:}

- Packing ratio.

- Property of surfactant.

- Property of oil phase.

- Temperature.

- Chain length.

- Nature of co-surfactant.

\section{CHARACTERISATION:}

\section{Phase Behaviour Studies}

- This study is very much essential for the determination of surfactant by using phase diagram which provide information on the boundaries of the different phases as the function of composition variables and temperature and more important structural organization can also be inferred.
- Phase behaviour studies also allow comparison of the efficiency of the different surfactant for given application.

- In this study, the temperature and pressure remain fixed $^{14}$.

\section{Scattering Technique}

- In this technique Small Angle X-ray Scattering (SAXS), Small Angle Neutron Scattering (SANS) and static as well as dynamic light scattering are widely applied technique in the study of microemulsion.

- In this technique, the intensity of scattered radiation $I(q)$ is maesured as a function of scattering vector $q$, $q=(4 \pi / \lambda) \sin \theta / 2$

$\theta=$ Scattering Angle.

$\lambda=$ Wavelength of Radiation.

- Small Angle X-ray Scattering technique and Static Light Scattering techniques both are used to determine the microemulsion droplet size and shape.

- Dynamic Light Scattering is used to analyse the fluctuation in the intensity of scattering by droplets due to Brownian motion ${ }^{15}$.

\section{Nuclear Magnetic Resonance Studies}

- The structure and dynamics of micro emulsion can be studied by using nuclear magnetic resonance techniques.

- Self diffusion measurements using different tracer technique generally radio labeling. Supply information on the mobility of the components.

\section{Electron Microscopic Studies}

- Microemulsion has been characterised by some electro microscopic techniques although the high lability of the sample and the possibilities of artifacts electron microscopy used to be considered a misleading techniques in microemulsion studies ${ }^{16}$.

5. Interfacial tension, Electrical conductivity and viscosity measurements: Formation and properties of micro emulsion can be studied by the measuring of Interfacial Tension. Ultra low values of Interfacial Tension are correleated with phase behaviour, particularly the existence of surfactant phase or middle phase micro emulsion in equilibrium with aqueous and oil phase.

- To determine the nature of the continuous phase and to detect phase inversion phenomena the electrical conductivity measurement are highly useful.

- Viscosity measurements can indicates the presence of rod like or warm like reverse micelles. It is used to determine the hydrodynamic radius of droplets, as well as interaction $\mathrm{b} / \mathrm{w}$ droplets and deviations from spherical shape by fitting the results to appropriate models ${ }^{17}$. 
Table 3: Evaluation parameters with technique used

\begin{tabular}{ll}
\hline PARAMETERS & TECHNIQUES USED \\
& \\
\hline Phase Behaviour & $\begin{array}{l}\text { Phase contrast microscopy \& freeze } \\
\text { fracture TEM }\end{array}$ \\
\hline Size and Shape & $\begin{array}{l}\text { Transmission Electron Microscopy (TEM), } \\
\text { SEM }\end{array}$ \\
\hline Rheology & Brookfield viscometer \\
\hline Conductivity & Conductivity meter \\
\hline Drug release studies & Franz Diffusion Cells \\
\hline Physical stability study & Ultracentrifuge \\
\hline
\end{tabular}

Table 4: Marketed lipid based and surfactant based Formulations

\begin{tabular}{lll}
\hline DRUG & PRODUCT & COMPONENT \\
\hline Cyclosporin & Neoral & $\begin{array}{l}\text { Corn oil mono-diglycerides, } \\
\text { cremophor RH40, ethanol, propylene } \\
\end{array}$ \\
& glycol, $\alpha$-tocopherol \\
\hline Ritonavir & Norvir & $\begin{array}{l}\text { Ethanol, oleic acid, cremophor EL, } \\
\text { BHT }\end{array}$ \\
\hline Progesterone & Prometrium & Peanut oil, lecithin, glycerin \\
\hline Doxecalciferol & Hectoral & $\begin{array}{l}\text { Medium chain triglycerides, ethanol, } \\
\end{array}$ \\
& & BHA \\
\hline
\end{tabular}

\section{APPLICATIONS:}

\section{Microemulsion as Drug Delivery Systems}

\section{1) Oral drug delivery-}

The most common method for drug delivery is through the oral route as it offers convenience and high patient compliance ${ }^{8}$.

\section{2) Parenteral drug delivery-}

Microemulsion systems intended for parenteral application have to be formulated using nontoxic and biocompatible ingredients. The oil in water microemulsion systems would be suitable to improve the solubility of poorly water soluble drug molecules whereas water in oil microemulsion systems would be best suited for optimizing the delivery of hydrophilic drug molecules that are susceptible to the harsh gastrointestinal condition.

\section{3) Ocular drug delivery-}

Aqueous solutions account for around $90 \%$ of the available ophthalmic formulations, mainly due to their simplicity and convenience However, extensive loss caused by rapid precorneal drainage and high tear turnover are among the main drawbacks associated with topical ocular drug delivery ${ }^{18}$.

\section{4)Topical drug delivery-}

A large amount of drug can be incorporated in the formulation due to the high solubilizing capacity that might increase thermodynamic activity towards the skin $^{7}$.

\section{5)Transdermal Drug Delivery-}

To the systemic circulation is one of the oldest routes that have been exploited using microemulsion systems ${ }^{19}$.

\section{CONCLUSION:}

From last 20 years a great progress has been made in understanding micro emulsion properties. In particular, interfacial film stability and micro emulsion structures can now be characterized in detail owing to the development of new and powerful techniques such as small-angle neutron scattering for better availability of drug delivery system.

- Microemulsions are optically isotropic and thermodynamically stable liquid solutions of oil, water and amphiphile.

- Microemulsions are readily distinguished from normal emulsions by their transparency, low viscosity and more fundamentally their thermodynamic stability.

- Drug delivery through microemulsions is a promising area for continued research with the aim of achieving controlled release with enhanced bioavailability and for drug targeting to various sites in the body.

\section{REFERENCES:}

1. Allen LV. Ansels Pharmaceutical Dosage forms and drug delivery systems. 8th edition.2005.

2. Alexander $T$ Florence and JuergenSiepmann. Modern Pharmaceutics, Vol.1( Drug and the pharmaceutical sciences vol.188) 5th ed., 2009 Informa health care.

3. Chauhan N, Kumar K, Pant NC. An updated review on transfersomes: a novel vesicular system for transdermal drug delivery. Universal Journal of Pharmaceutical Research 2017; 2(4): 42-45.

4. Agarwal P, Semimul A. A comprehensive review on sustained release matrix tablets: a promising dosage form. Universal Journal of Pharmaceutical Research 2018; 3(6): 49-54.

5. Aulton, Michael E. (Editor) Aulton's Pharmaceutics: The Design and Manufacture of Medicines (3rd ed.). Churchill Livingstone. 2007;384:390-405.

6. ALGIN YAPAR E, BESKAN U, KARAVANA SY. A recent overview of locally administered topical otic dosage forms. Universal Journal of Pharmaceutical Research 2019; 4(4): 39-42.

7. Ikechukwu UR, John Francis DE, Ambi AA. Development and evaluation of Ritonavir hollow microballoons for floating drug delivery. Universal Journal of Pharmaceutical Research 2017; 2(2): 811.

8. Khan AY, Talegaonkar S, Iqbal Z, Ahmed FJ, Khar RK. Multiple emulsions: an overview. Curr Drug 
Deliv. 2006;3(4):429-43.http://dx.doi.org/ 10.2174 /15672010 6778559056;PMid:17076645.

9. Yusuf FS. Formulation and in-vitro evaluation of floating microballoons of stavudine. Universal Journal of Pharmaceutical Research 2016; 1(1): 811.

10. Yusuf FS, Yunus AA, John DF, Chigbo UJ. Abacavir loaded nanoparticles: preparation, physicochemical characterization and in vitro evaluation. Universal Journal of Pharmaceutical Research 2016; 1(2): 11-14.

11. Kumar P, Mittal KL. Handbook of Micro emulsion Science and Technology; 1st Edn; CRC Press, New York,1999.

12. Peter OI, Ifeoma UC. Development and evaluation of Albendazole microcapsule for colonic drug delivery system. Universal Journal of Pharmaceutical Research 2017; 2(2): 4-7.

13. Nweje-Anyalowu Paul C, Anyalogbu Ernest AA, White Alalibo Jim. Design and evaluation of chronotherapeutic pulsatile drug delivery system of Cilnidipine. Universal Journal of Pharmaceutical Research 2017; 2(5): 15-18.

14. Tang JL, Sun J, He ZG. Self-Emulsifying Drug Delivery Systems: Strategy For Improving Oral
Delivery of Poorly Soluble Drugs. Current Drug Therapy.2007;2(1):85-93.http://dx.doi.org/10.2174 /157488507779422400.

15. Kaur G, Paliwal S. Formulation and evaluation of etoricoxib microbeads for sustained drug delivery. Universal Journal of Pharmaceutical Research 2019; 4(1): 35-39.

16. Sunday OS. Colon-targeted drug delivery systems: design, trends and approaches. Universal Journal of Pharmaceutical Research 2017; 2(4): 46-50.

17. Talegaonkar S, Azeem A, Ahmad FJ, Khar RK, Pathan SA, Khan ZI. Microemulsions: A Novel Approach to Enhanced Drug Delivery. Recent Patents on Drug Delivery \& Formulation. 2008;2(3): 238-257.http://dx.doi. org/10.2174/187221108786241679.

18. Min DI. Neoral: a micro emulsion cyclosporine. J Transpl Coord Review. Erratum in: J Transpl Coord. 1996;6(2):52.http://dx.doi.org/10.7182/prtr.1.6.1.f 04016025 h h795up;PMid:9157923.

19. Tenjarla S. Microemulsions: an overview and pharmaceutical applications. Crit RevTher Drug Carrier Syst.1999;16(5):461-521.http:// dx.doi.org/ 10.1615/critrevtherdrugcarriersyst.v16.i5.20. 